\title{
A Discontinuity Adaptive Prior for Image Denoising
}

\author{
Aravind $\mathrm{B} \mathrm{N}$ \\ Kalpataru Institute of Technology \\ Tiptur - 572202 \\ Karnataka, India
}

\author{
K V Suresh \\ Siddaganga Institute of Technology \\ Tumkur -572103 \\ Karnataka, India
}

\begin{abstract}
The presence of noise in digital images degrades the visual quality by corrupting the information associated with the image. The aim of denoising is to restore an image from its noisy version by preserving signal information. In this paper, we are considering an image corrupted by additive Gaussian noise. The image is modeled as Markov random field (MRF) and an estimation of maximum-a-posteriori (MAP) is obtained using graduated non-convexity. The results are compared with other spatial domain filtering methods. The discontinuity adaptive prior helps in preserving edge information. The results suggest that proposed method has an improved performance.
\end{abstract}

\section{Keywords}

Image denoising, Markov random field, Discontinuity adaptive, Graduated non-convexity.

\section{INTRODUCTION}

Analysis of an image in the presence of noise will be difficult. The noise may be due to non-linearity of capturing device, poor lighting and/or other atmospheric conditions. Restoring such images is a challenging issue. The main aim of any denoising algorithm is to suppress the noise while retaining details in the image. It is difficult to attain this objective. Many denoising methods have been developed over past decades which can be grouped into two basic categories: transform domain [1-5] and spatial domain [6-9][11]. While, spatial domain methods use direct pixel values for denoising, transform domain methods project image onto a set of bases and process the coefficients. Research depicts that both approaches are being used extensively in denoising. This paper aims at developing edge preserving spatial domain method of denoising.

The degradation function in spatial domain is represented as,

$$
y=x * h+\eta
$$

where $y$ is degraded image, $h$ is degradation function, $x$ is original image, $\eta$ is additive noise and $*$ is the convolution operator [12]. In this paper degradation due to noise is considered. Hence Equation (1) takes the form,

$$
y=x+\eta
$$

where, $\eta$ is considered as Gaussian noise.

\section{SPATIAL DOMAIN DENOISING}

Methods where the pixel intensities are used directly in the denoising process are said to be spatial domain filters. Almost all spatial domain methods perform denoising locally considering neighboring pixels into account. Local mean and variance are used as the basis for making changes that depend on image characteristics in a predefined region about each pixel in the image. Local mean and variance is given by [7]

$$
\begin{gathered}
m_{i, j}=\frac{1}{(2 m+1)(2 n+1)} \sum_{k=i-n}^{n+i} \sum_{l=j-m}^{j+m} y_{k, l} \\
v_{i, j}=\frac{1}{(2 m+1)(2 n+1)} \sum_{k=i-n}^{n+i} \sum_{l=j-m}^{j+m}\left(y_{k, l}-m_{i, j}\right)^{2}
\end{gathered}
$$

where $m_{i, j}$ and $v_{i, j}$ are mean and variance w.r.t $(i, j)$.

Lee [8] extended the idea of [7] by defining a priori mean and variance. A priori mean $\widehat{m}_{i, j}$ is same as Equation (3) and variance is given by;

$$
\hat{v}_{i, j}=\frac{1}{(2 m+1)(2 n+1)} \sum_{k=i-n}^{n+i} \sum_{l=j-m}^{j+m}\left(y_{k, l}-\widehat{m}_{i, j}\right)^{2}-\sigma_{n}^{2}(5)
$$

Now, final estimated image $\hat{x}_{i, j}$ is computed by

$$
\hat{x}_{i, j}=\widehat{m}_{i, j}+k_{i, j}\left(y_{i, j}-\widehat{m}_{i, j}\right)
$$

where $k_{i, j}$ is gain and is given by;

$$
k_{i, j}=\frac{\hat{v}_{i, j}}{\hat{v}_{i, j}+\sigma_{1}^{2}}
$$

$\sigma_{1}^{2}$ is a constant.

For low signal to noise ratio, $\hat{v}_{i, j}$ is small compared to $\sigma_{1}^{2}$, $k_{i, j} \cong 0$ and the estimated $\hat{x}_{i, j}$ is $\hat{m}_{i, j}$. For a high signal to noise ratio, $\hat{v}_{i, j}$ is much larger than $\sigma_{1}^{2}, k_{i, j} \cong 1$ and $\hat{x}_{i, j} \cong$ $y_{i, j}$.

Lee filter considers that all pixel values with in a local window are almost same [9]. A weighted form of Equation (5) given as [10];

$$
v_{i, j}=\sum_{k=i-n}^{i+n} \sum_{l=j-m}^{j+m} w(i, j, k, l)\left[y_{k, l}-m_{i, j}\right]^{2}
$$

Jin et al., [9] proposed an adaptive approach to select $w(\cdot)$.

$$
w(i, j, k, l)=\frac{K(i, j)}{1+a\left\{\max \left[E^{2},(y(i, j)-y(k, l))^{2}\right]\right\}}
$$

where $K(i, j)$ is gain and is given by,

$$
K(i, j)=\left\{\sum_{p, q} \frac{1}{1+a\left\{\max \left[\varepsilon^{2},(y(i, j)-y(p, q))^{2}\right]\right\}}\right\}^{-1}
$$

where $a(a>0)$ and $\varepsilon$ are the parameters of weight function. Using the weight function in Equation (9), [9] realizes local mean and variance adaptively as, 


$$
\begin{gathered}
\hat{m}_{i, j}=\sum_{k=i-n} \sum_{l=j-m}^{i+n} w(i, j, k, l) y(k, l) \\
\hat{v}_{i, j}=\sum_{k=i-n}^{j+m} \sum_{l=j-m}^{j+m} w(i, j, k, l)[y(k, l)-\widehat{m}(i, j)]^{2}
\end{gathered}
$$

Bilateral filtering is a non-linear filter proposed by Tomasi and Manduchi [11]. It is non-iterative and local approach to edge preserving smoothing. The gray level value at each pixel is calculated by considering the geometric and photometric similarities between neighboring pixels with in spatial window. In discrete form, bilateral filtering is given as;

$$
I^{*}(\vec{x})=\frac{1}{k(\vec{x})} \sum_{\vec{\xi} \in N_{s}} I(\vec{\xi}) \cdot c(\vec{\xi}, \vec{x}) \cdot s(I(\vec{\xi}), I(\vec{x}))
$$

where $\vec{x}$ and $\vec{\xi}$ are spatial coordinates. I is noisy image, $I^{*}$ is filtered image, $N_{s}$ is spatial window, $k(\vec{x})$ is the normalization constant which assures the weights $c(\cdot) \cdot s(\cdot)$ are added up to 1 with in $N_{s} . c$ and $s$ are measures of geometric and photometric similarities between neighborhoods respectively.

Bilateral filtering is a non-iterative method only if a wide spatial window is used. A wide spatial window may over smooth sharp edges. Therefore, it is necessary to make a balance between the size of spatial window and the number of iterations [13] to be performed in bilateral filtering. Trilateral filter [14] considers not only the geometric and photometric similarities, but also the local structural similarity to smooth the images with a narrow spatial window while preserving edges. It applies lowpass filter for homogeneous regions. Smoothing along edges is achieved by considering geometric, photometric and local structural similarities between neighboring pixels.

Rudin and Osher [15] introduced Total Variation (TV) minimization for continuous signals. Later its discrete version was developed by Chen et al., [16]. Graphs and edge derivatives are used to find edges. Digital TV filter contains two parameters;

- a small positive parameter $a$ called the regularization parameter.

- a positive number $\lambda$ called fitting parameter.

TV filtering at location $\alpha$ has following steps;

(i) Compute local variation $|\nabla u|_{\alpha}$ at $\alpha$ and all its neighbourhoods $\beta$.

(ii) Compute the weights $w_{\alpha, \beta}$ using

$$
w_{\alpha, \beta}(u)=\frac{1}{\left|\nabla_{\alpha} u\right|_{\alpha}}+\frac{1}{\left|\nabla_{\beta} u\right|_{\alpha}}
$$

(iii)Compute the filter coefficients $h_{\alpha \alpha}$ and $h_{\alpha \beta}$;

$$
\begin{gathered}
h_{\alpha \beta}=\frac{w_{\alpha \beta}(u)}{\lambda+\sum_{\gamma \sim \alpha} w_{\alpha \gamma}(u)} \\
h_{\alpha \alpha}=\frac{\lambda}{\lambda+\sum_{\gamma \sim \alpha} w_{\alpha \gamma}(u)}
\end{gathered}
$$

(iv)Filtering $F_{\alpha}(u)=\sum_{\gamma \sim \alpha} h_{\alpha \beta} u_{\beta}+h_{\alpha \alpha} u_{\alpha}^{0}$

$\mathrm{TV}$ is an iterative process. The parameter $\lambda$ controls the regularity and fidelity terms. As $\lambda$ gets smaller, the weight of the regularity term increases. Also, details and texture will get oversmoothed [17].

Non-local means (NLM) is one of the state-of-the-art denoising methods proposed by Buades et al., [6], [17 - 19]. The basic idea is that, an image consists of repeated structures and averaging them will reduce the noise. At location $i$, the estimated value $\mathrm{NL}[\mathrm{y}](\mathrm{i})$ is computed as a weighted average of all pixels in the image;

$$
N L[y](i)=\sum_{j \in I} w(i, j) y(j)
$$

where the family of weights $\{w(i, j)\}_{j}$ depends on the similarity between the pixels $i$ and $j$, and satisfy the conditions $0 \leq w(i, j) \leq 1$ and $\sum_{j} w(i, j)=1$.

Similarity between two pixels $i$ and $j$ depends on the similarity of intensity gray level vectors $v\left(N_{i}\right)$ and $v\left(N_{j}\right)$, where $N_{k}$ denotes a square neighbourhood centered at $k$. The weight function is defined as,

$$
w(i, j)=\frac{1}{z(i)} e^{-\frac{\left\|v\left(N_{i}\right)-v\left(N_{j}\right)\right\|_{2, a}^{2}}{h^{2}}}
$$

where $z(i)$ is the normalizing constant defined as,

$$
z(i)=\sum_{j} e^{-\frac{\| v\left(N_{i}\right)-\left.v\left(N_{j}\right)\right|_{2, a} ^{2}}{h^{2}}}
$$

and the parameter $h$ acts as the degree of filtering.

For an image with $M$ pixels, $M$ weights have to be computed at each pixel. Hence a total of $M^{2}$ weights make the algorithm computationally intensive [20]. Also, this approach is suitable for denoising images with periodic texture patterns, but fails for severely degraded image due to the corruption of image structures. In most of the denoising algorithms there is always a trade-off between denoising methods and edge preservation. In this work, an attempt is made to model the image as MRF with a discontinuity adaptive prior, which helps in better edge preservation.

\subsection{Markov Random Field}

Markov random field models provide a unified framework for many computer vision tasks. For problems in low-level vision, probabilistic prior models of the spatial structure of images or scene properties are often formulated as Markov random fields [21 - 25]. Markov random fields have found many areas of application including image denoising [26], stereo imaging [27], optical flow estimation [28] and texture classification [29], to name a few.

MRF is a two-dimensional (2D) random process defined on a discrete lattice. Usually a lattice is a regular grid on the 2D plane. They yield flexible stochastic image models describing the intensity distribution in images. The main advantage is that, one can obtain a global representation of the image by means of local conditional probability distributions. This makes MRF quite attractive in several image processing applications including image restoration [22].

A random field $F=\left\{F_{i j}\right\}$ designed over lattice $\mathcal{S}$ is a Markov random field considering the neighborhood system $\mathcal{N}$, iff

- $P(F=f)>0, \forall f \in \mathbb{F}$ (positivity)

- $P\left[F_{i j}=f_{i j} \mid F_{k l}=f_{k l} \forall(k, l) \neq(i, j)\right]=P\left[F_{i j}=f_{i j} \mid F_{k l}=\right.$ $\left.f_{k l},(k, l) \in \mathcal{N}_{i j}\right]$ (Markovianity)

for all $(\boldsymbol{i}, \boldsymbol{j}) \in \boldsymbol{S}$. Here, $\boldsymbol{f}$ is realization of random field $\boldsymbol{F}$ and $\mathbb{F}$ represents the configuration space.

\subsection{Gibbs Random Field}

Gibbs random field $F=\left\{F_{i j}\right\}$ over neighbourhood system $\mathcal{N}$ is defined by joint distribution function 


$$
P(F=f)=\frac{1}{Z} \exp \{-U(f)\}
$$

where $U(f)=\sum_{c \in C} V_{c}(f)$ and $Z=\sum_{f \in \mathbb{F}} \exp \{-U(f)\}$

The distribution given in Equation(20) is called the Gibbs distribution (GD). The term $U(f)$ (energy function) is associated with cliques and $Z$ is partition function. $V_{c}(f)$ is clique potential and its careful selection decides the power of MRF. GRF is characterized by global property, whereas, MRF represents local property. A theorem by Hammersly and Clifford [30] established an equivalence relation between GRF and MRF. This made MRF to be used for image representation.

\section{PROBLEM FORMULATION}

To solve for Equation (2), a priori information about $x$ is important and it can be accomplished by Bayesian MAP formulation. The MAP estimate of the denoised image $x$ is given by,

$$
\hat{x}=\arg \max _{x}\{P(x \mid y)\}
$$

Using Bayes' rule, this can be written as,

$$
\hat{x}=\arg \max _{x}\left\{\frac{p(y \mid x) p(x)}{p(y)}\right\}
$$

or equivalently,

$$
\hat{x}=\arg \max _{x}\{p(y \mid x) p(x)\}
$$

Taking the logarithm of the posteriori probability, the MAP estimate of $x$ is given by,

$$
\hat{x}=\arg \max _{x}\{\log [P(y \mid x)]+\log [P(x)]\}
$$

From MRF-Gibbs equivalence [30],

$$
P[X=x]=\frac{1}{Z} \exp \{-U(x)\}=\frac{1}{Z} \exp \left\{-\sum_{c \in C} V_{c}(x)\right\}
$$

Therefore,

$\hat{x}=\arg \max _{x}\left\{\log [P(y \mid x)]+\log \left[\frac{1}{Z} \exp \left\{-\sum_{c \in C} V_{c}(x)\right\}\right]\right\}$

Using the observation model; $y=x+\eta$; and the fact that the noise fields are statistically independent of $X$ and as well as each other, we have,

$$
P[Y=y \mid X=x]=\frac{1}{\left(2 \pi \sigma^{2}\right)^{\frac{M_{1} M_{2}}{2}}} \exp \left[-\frac{\|y-x\|^{2}}{2 \sigma^{2}}\right]
$$

where $\sigma_{n}^{2}$ is the noise variance.

Using equation Equation (25) and Equation (26), we get,

$$
\hat{x}=\arg \min _{x}\left\{\frac{\|y-x\|^{2}}{2 \sigma^{2}}+\sum_{c \in C} V_{c}(x)\right\}
$$

We are using discontinuity adaptive (DA) model where the discontinuities are preserved by adaptively adjusting the interaction between pixels across edges. Using DA prior function of [31], considering second order neighborhood for MRF,

$$
\begin{array}{rl}
\sum_{c \in C} V_{c}(x)=\sum_{i=1} \sum_{j=1}^{N_{1}} & 8 \gamma-\gamma \exp \left\{-\frac{[x(i, j)-x(i, j-1)]^{2}}{\gamma}\right\} \\
& -\gamma \cdot \exp \left\{-\frac{[x(i, j)-x(i, j+1)]^{2}}{\gamma}\right\} \\
& -\gamma \cdot \exp \left\{-\frac{[x(i, j)-x(i-1, j)]^{2}}{\gamma}\right\} \\
& -\gamma \cdot \exp \left\{-\frac{[x(i, j)-x(i+1, j)]^{2}}{\gamma}\right\} \\
& -\gamma \cdot \exp \left\{-\frac{[x(i, j)-x(i-1, j-1)]^{2}}{\gamma}\right\} \\
& -\gamma \cdot \exp \left\{-\frac{[x(i, j)-x(i-1, j+1)]^{2}}{\gamma}\right\} \\
& -\gamma \cdot \exp \left\{-\frac{[x(i, j)-x(i+1, j-1)]^{2}}{\gamma}\right\} \\
& -\gamma \cdot \exp \left\{-\frac{[x(i, j)-x(i+1, j+1)]^{2}}{\gamma}\right\}
\end{array}
$$

Using Equation (28) in Equation (27) and finding the gradient at the $n^{\text {th }}$ iteration, we get

$$
\operatorname{grad}^{(n)}=\frac{1}{\sigma_{n}^{2}} \sum_{r=1}^{m}\left(x-y_{r}\right)+\lambda G^{(n)}
$$

where, $\lambda$ is regularization parameter and $G$ is Gradient at position $(i, j)$.

$G^{(n)}(i, j)$

$=2[x(i, j)-x(i, j-1)] \exp \left\{-\frac{[x(i, j)-x(i, j-1)]^{2}}{\gamma}\right\}$

$+2[x(i, j)-x(i, j-1)] \exp \left\{-\frac{[x(i, j)-x(i, j+1)]^{2}}{\gamma}\right\}$

$+2[x(i, j)-x(i, j-1)] \exp \left\{-\frac{[x(i, j)-x(i-1, j)]^{2}}{\gamma}\right\}$

$+2[x(i, j)-x(i, j-1)] \exp \left\{-\frac{[x(i, j)-x(i+1, j)]^{2}}{\gamma}\right\}$

$+2[x(i, j)-x(i, j-1)] \exp \left\{-\frac{[x(i, j)-x(i-1, j-1)]^{2}}{\gamma}\right\}$

$+2[x(i, j)-x(i, j-1)] \exp \left\{-\frac{[x(i, j)-x(i-1, j+1)]^{2}}{\gamma}\right\}$

$+2[x(i, j)-x(i, j-1)] \exp \left\{-\frac{[x(i, j)-x(i+1, j-1)]^{2}}{\gamma}\right\}$

$+2[x(i, j)-x(i, j-1)] \exp \left\{-\frac{[x(i, j)-x(i+1, j+1)]^{2}}{\gamma}\right\}$

More details about DAMRF and deterministic annealing are presented in [32-33].

\section{RESULTS AND DISCUSSION}

In this section, comparison between proposed method and other spatial domain techniques is presented. For quantitative analysis PSNR is used as benchmark;

$$
P S N R=10 \log _{10}\left(\frac{255^{2}}{M S E}\right)
$$

where, MSE is the mean square error.

For simulation three test images were considered; Lena $(512 \times 512)$, Boat $(256 \times 256)$ and Child $(256 \times 256)$. Noisy versions of the images were obtained by adding Gaussian noise of different variance values. Figure 1 shows the denoising of Lena image degraded by $\sigma=20$ (Figure 1(a)). Denoising is achieved by using various spatial domain methods. Figure $1(\mathrm{~b})-(\mathrm{g})$ are the resultant images. First, the 
image is denosied by arithmetic and geometric mean filters of mask size $3 \times 3$ and results are presented in Figure 1(b) and (c) respectively. Geometric mean reduces noise to a smaller extent and creates visual artifacts. Arithmetic mean filter smoothens the noise and also edges. Result of $3 \times 3$ adaptive Wiener filter is shown in Figure 1(d). It provides better performance compared to mean filters. Further improvement is observed in $5 \times 5$ adaptive Wiener filter (Figure 1(e)). Bilateral filtering [34] (Figure 1(f)) provides over smoothed representation. Figure $1(\mathrm{~g})$ is the resultant of proposed DAMRF method. Reconstructed image has better smoothing and edges are well preserved. Figure 2 shows the result of Child image degraded by $\sigma=30$. The proposed method provides better results with negligible amount of artifacts. (Figure 2(g)).

Table 1 shows the quantitative analysis of various denoising methods. All denoising methods provide better results at low noise levels. As the noise increases, PSNR value of proposed method remains higher. MSE and PSNR estimate perceived errors; on the other hand, a quality measure called structural similarity (SSIM) index [35] considers image degradation as perceived change in structural information. Table 2 provides mean SSIM of various denoising methods. In Table 1, even though PSNR of additive Wiener filter is better than proposed method, MSSIM clearly indicates that the proposed method yields better than other methods.

Next, experiment is conducted on real "Traffic signal" images of size $480 \times 640$ captured at different weather conditions.
Noise estimation is done using absolute median filter of [1]. Denoising is achieved using bilateral filter and proposed method. Figure 3(a) shows the noisy traffic signal image. Figure 3(b) is the image denoised by bilateral filter. Result of proposed method is presented in Figure 3(c). For better visualization, text region of Figure 3(a)-(c) is zoomed and shown in Figure 4(a)-(c) respectively. It can be observed that, the quality of text reconstruction is better in proposed method. The letter ' $a$ ' is over smoothed in bilateral filter but the proposed method yields a good reconstruction. The same is repeated for another traffic signal image and results are provided in Figure 5.

\section{CONCLUSION}

In this paper, an edge preserving MAP-MRF technique is proposed for image denoising. Images corrupted with Gaussian noise of different variance values are considered for experimentation. Simulations of denoising using mean filters, adaptive Wiener filter, bilateral filter and proposed DAMRF methods are given for both synthetic and real images. Results show that, the proposed method provides a better performance compared to other techniques by suppressing the noise and preserving edges.

In this paper, the noise is assumed to be additive Gaussian. Image denoising under non-Gaussian type of noise could be explored. Further, the work can be extended using a combination of spatial and transform domain techniques for improved performance.

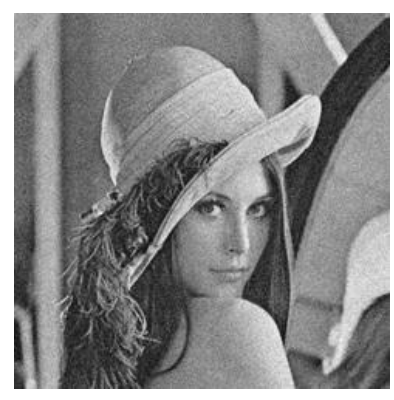

(a)

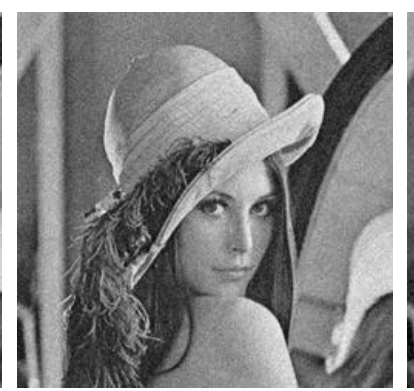

(b)

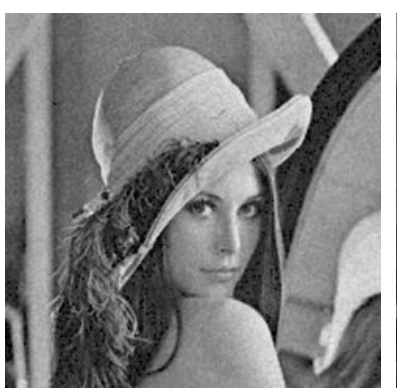

(c)

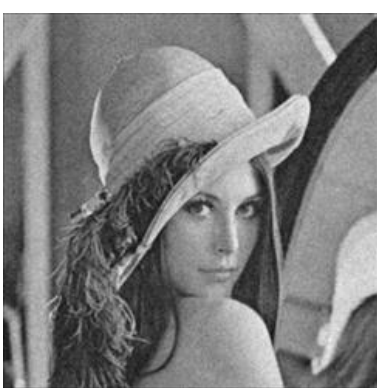

(d)

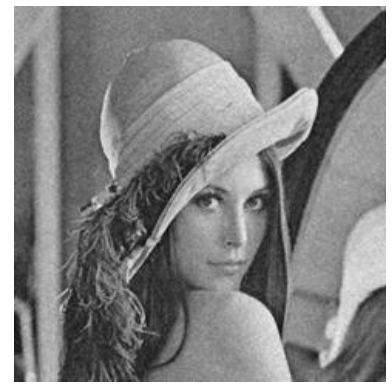

(e)

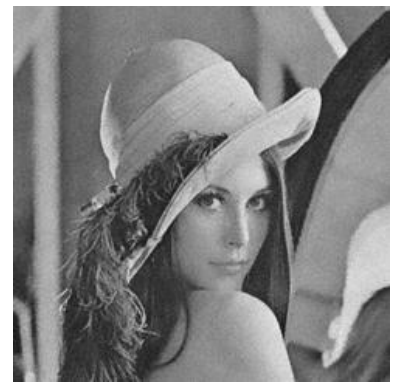

(f)

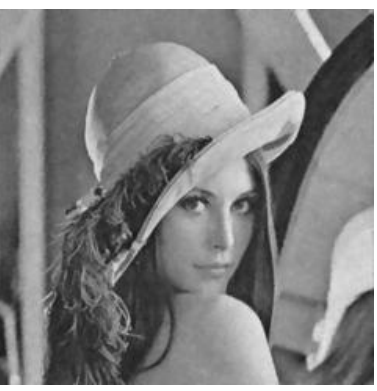

(g)

Fig. 1 : Result of various filtering methods in spatial domain. (a) Noisy image $(\sigma=20)$ (b) Arithmetic mean filter (c) Geometric mean filter (d)-(e) Adaptive Wiener filter (3x3) and (5x5) respectively (f) Bilateral filtering (g) Proposed method. 


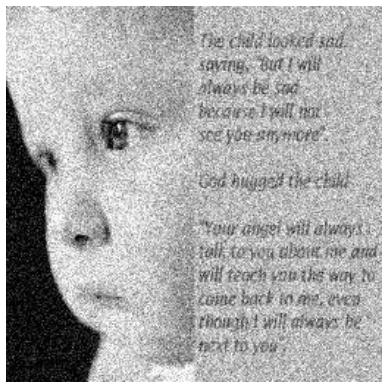

(a)

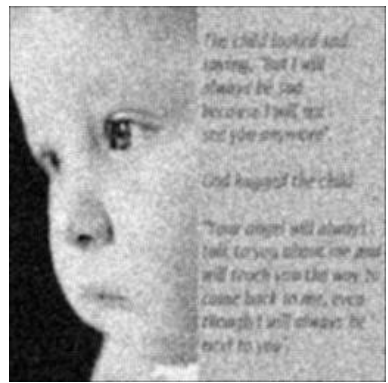

(b)

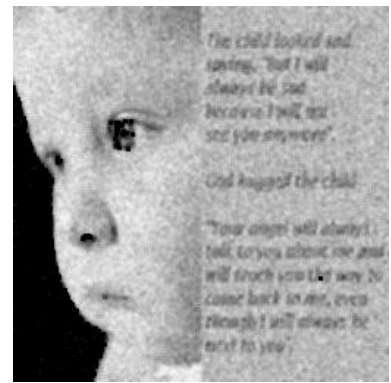

(c)

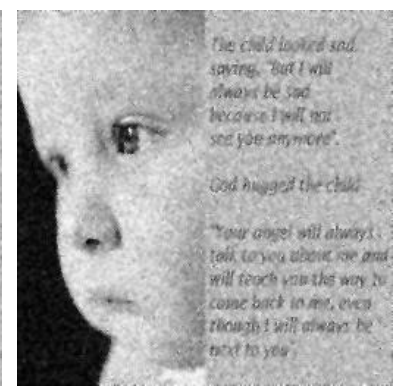

(d)

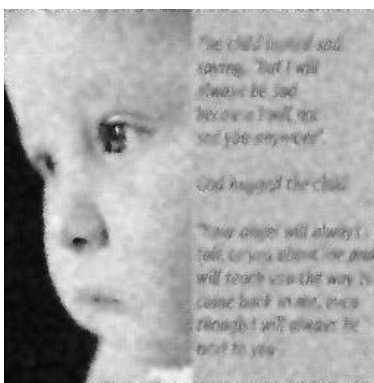

(e)

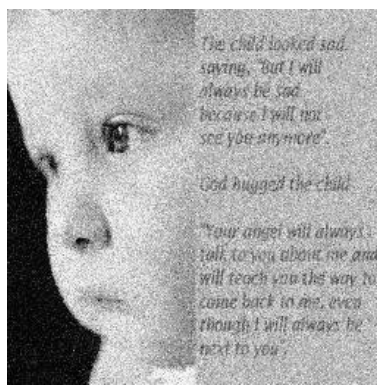

(f)

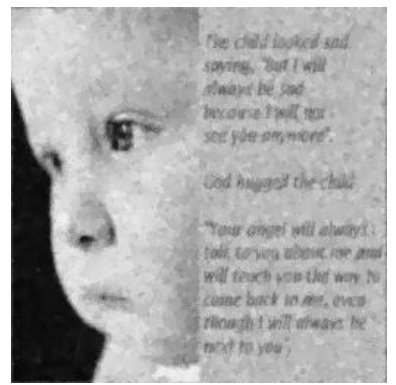

(g)

Fig. 2: Result of various Filtering methods in spatial domain. (a) Noisy image $(\sigma=30)$ (b) Arithmetic mean filter (c) Geometric mean filter (d)-(e) Adaptive Wiener filter (3x3) and (5x5) respectively. (f) Bilateral filter (g) Proposed method.

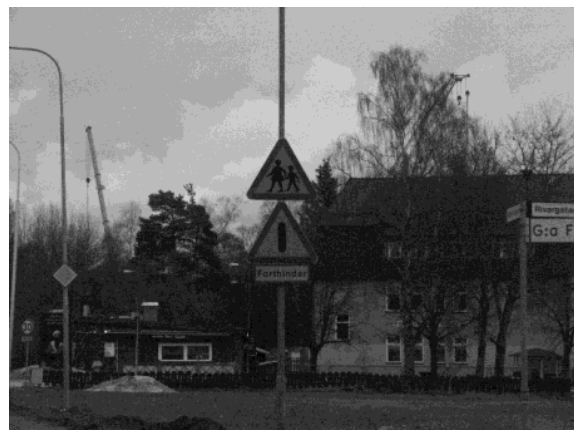

(a)

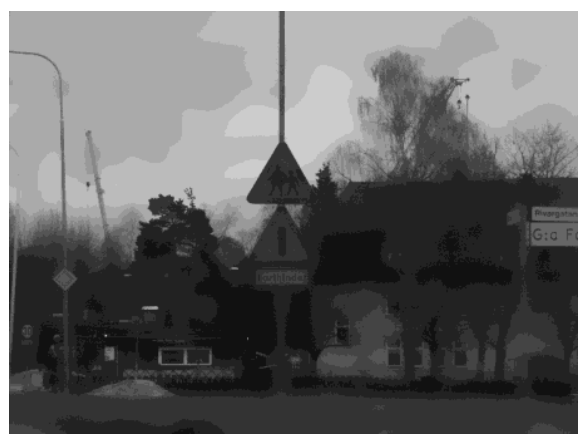

(b)

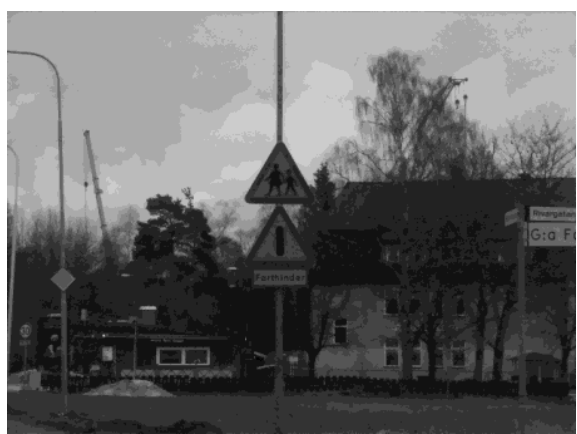

(c)

Fig. 3: Denoising of traffic signal image using various filtering methods. (a) Noisy traffic signal image. (b) Bilateral filtering (c) Result of proposed method. (Traffic signal images are the courtesy of Dr. Hasan Fleyeh, Dalarna University, Sweden)

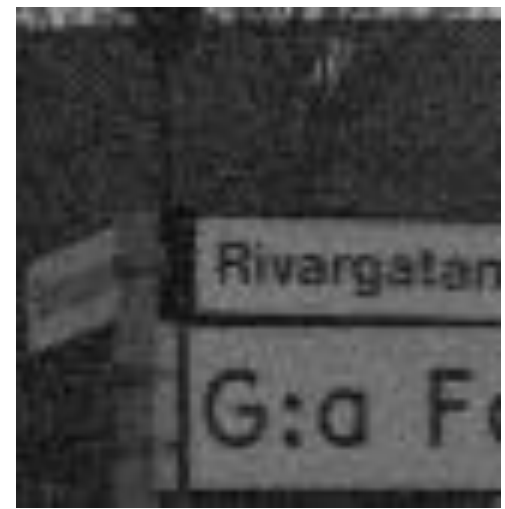

(a)

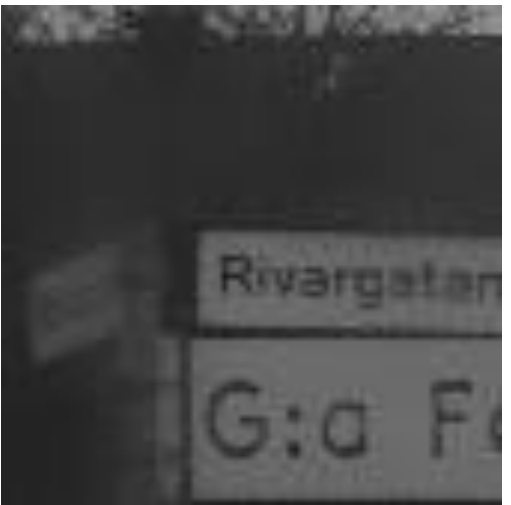

(b)

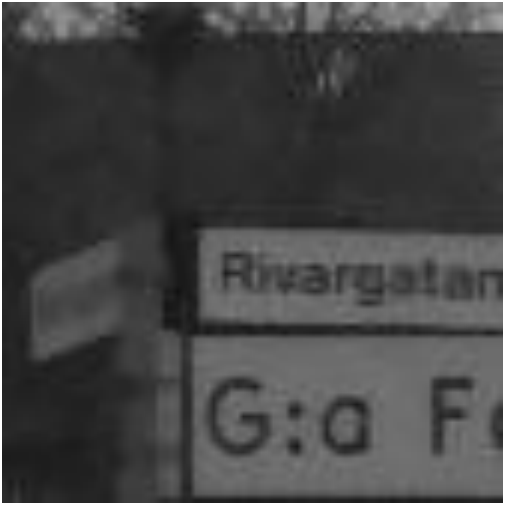

(c)

Fig. 4: Zoomed text region of Figure 3(a) - (c) respectively. 


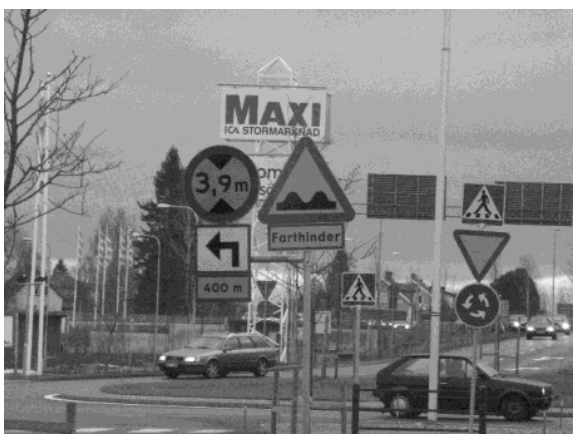

(a)

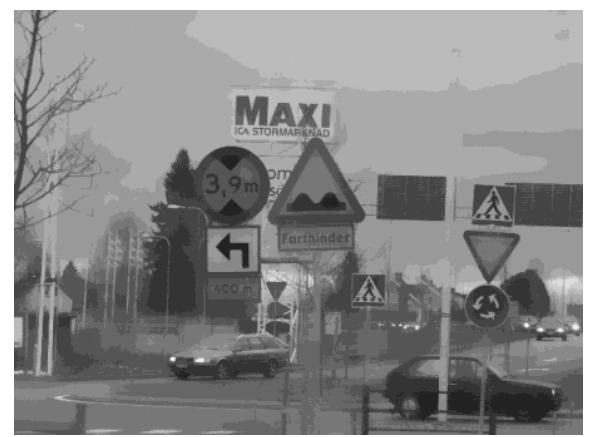

(b)

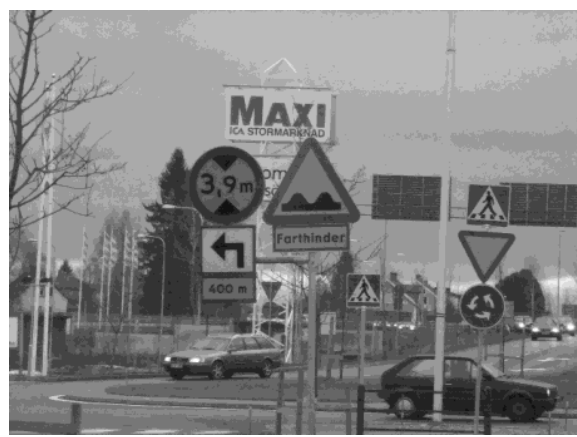

(c)

Figure 5: Denoising of traffic signal image using various filtering methods. (a) Noisy traffic signal image. (b) Bilateral filtering (c) Result of proposed method.

Table 1: Quantitative analysis of various filtering methods

\begin{tabular}{|c|c|c|c|c|c|c|c|}
\hline \multirow[b]{2}{*}{$\sigma$} & \multicolumn{7}{|c|}{ PSNR (dB) } \\
\hline & $\begin{array}{l}\text { Arithmetic } \\
\text { mean }(3 \times 3)\end{array}$ & $\begin{array}{l}\text { Geometric } \\
\text { mean }(3 \times 3)\end{array}$ & $\begin{array}{c}\text { Adaptive } \\
\text { Wiener filter } \\
(\mathbf{3 x} 3)\end{array}$ & $\begin{array}{c}\text { Adaptive } \\
\text { Wiener filter } \\
(5 \times 5)\end{array}$ & $\begin{array}{l}\text { Bilateral } \\
\text { filter }\end{array}$ & $\begin{array}{l}\text { Proposed } \\
\text { method }\end{array}$ & Image \\
\hline 10 & 30.9030 & 31.9498 & 33.5354 & 32.6329 & 31.5130 & 32.6443 & \multirow{3}{*}{$\begin{array}{c}\text { Lena }(512 \times \\
512)\end{array}$} \\
\hline 20 & 28.7975 & 27.4381 & 28.9987 & 29.9300 & 25.7036 & 29.974 & \\
\hline 30 & 26.7016 & 22.6441 & 25.8540 & 27.8587 & 21.0037 & 28.2728 & \\
\hline 10 & 25.9493 & 26.8948 & 32.0521 & 29.8316 & 32.0162 & 33.5546 & \multirow{3}{*}{$\begin{array}{c}\text { Child }(256 \times \\
256)\end{array}$} \\
\hline 20 & 25.1721 & 25.6652 & 27.9789 & 27.5130 & 25.9231 & 28.7293 & \\
\hline 30 & 24.1027 & 24.0928 & 25.0954 & 25.5882 & 21.2698 & 25.8390 & \\
\hline 10 & 28.4742 & 31.1189 & 32.8666 & 31.5788 & 30.3045 & 31.3422 & \multirow{3}{*}{$\begin{array}{c}\text { Boat }(256 \times \\
256)\end{array}$} \\
\hline 20 & 27.1316 & 28.0828 & 28.8236 & 29.1234 & 25.3543 & 27.9032 & \\
\hline 30 & 25.6124 & 24.0382 & 25.7482 & 27.2404 & 20.8797 & 26.2419 & \\
\hline
\end{tabular}

Table 2: Structural similarity based image quality assessment [35]

\begin{tabular}{|c|c|c|c|c|c|c|c|}
\hline \multirow[b]{2}{*}{$\sigma$} & \multicolumn{7}{|c|}{ MSSIM } \\
\hline & $\begin{array}{l}\text { Arithmetic } \\
\text { mean }(3 \times 3)\end{array}$ & $\begin{array}{l}\text { Geometric } \\
\text { mean }(3 \times 3)\end{array}$ & $\begin{array}{c}\text { Adaptive } \\
\text { Wiener filter } \\
(3 \times 3)\end{array}$ & $\begin{array}{c}\text { Adaptive } \\
\text { Wiener filter } \\
(5 \times 5)\end{array}$ & $\begin{array}{l}\text { Bilateral } \\
\text { filter }\end{array}$ & $\begin{array}{l}\text { Proposed } \\
\text { method }\end{array}$ & Image \\
\hline 10 & 0.9377 & 0.9363 & 0.9421 & 0.9416 & 0.9311 & 0.9491 & \multirow{3}{*}{$\begin{array}{c}\text { Lena }(512 \times \\
512)\end{array}$} \\
\hline 20 & 0.8385 & 0.8102 & 0.8263 & 0.8913 & 0.8132 & 0.9095 & \\
\hline 30 & 0.7344 & 0.6557 & 0.7057 & 0.8224 & 0.6257 & 0.8651 & \\
\hline 10 & 0.8091 & 0.7665 & 0.8929 & 0.8852 & 0.8930 & 0.9291 & \multirow{3}{*}{$\begin{array}{c}\text { Child }(256 \times \\
256)\end{array}$} \\
\hline 20 & 0.6803 & 0.6358 & 0.7344 & 0.8066 & 0.5539 & 0.8548 & \\
\hline 30 & 0.5582 & 0.5182 & 0.5706 & 0.7073 & 0.3374 & 0.6858 & \\
\hline 10 & 0.9334 & 0.9246 & 0.9450 & 0.9129 & 0.9118 & 0.9459 & \multirow{3}{*}{$\begin{array}{c}\text { Boat }(256 \times \\
256)\end{array}$} \\
\hline 20 & 0.8542 & 0.8152 & 0.8555 & 0.8710 & 0.8315 & 0.8827 & \\
\hline 30 & 0.7640 & 0.6971 & 0.7480 & 0.8143 & 0.6723 & 0.8254 & \\
\hline
\end{tabular}

\section{REFERENCES}

[1] D.L. Donoho. 1995. Denoising by soft threhsolding. IEEE Trans. on Information Theory. (May 1995), 613627.

[2] R.R. Coifman and D.L. Donoho. 1995. Translation invariant denoising. Wavelet and Statistics, Springer
Lecture Notes in Statistics, Springer-Verlag. (1995), 125 150 .

[3] T. Tony cai and Bernard W. Silverman. 2001. Incorporating information on neighbouring wavelet coefficients. Sankhya. (Ser. B63 2001), 127-148, 2001. 
[4] Zhou dengwen and Cheng Wengang. 2008. Image denoising with an optimal threhsolding and neighbouring window. Pattern Recognition Letters, Elsevier. (Aug. 2008), 1694-1697.

[5] D. Cho, T.D. Bui and G. Chen. 2009. Image denoising based on wavelet shrinkage using neighbour and level dependency. International Journal of Wavelets, Multiresolution and Information Processing. (2009), 299311.

[6] Antoni Buades, Bartoneu Coll and Jean Michel Morel. 2008. Non-local image and movie denoising. International Journal of Computer Vision, Springer. (Feb 2008), 123-139.

[7] R. Wallis. 1976. An approach to the space variant restoration and enhancement of images. Symposium on Current Mathematical Problems in Image Science, Naval postgraduate school, Monterey, CA.

[8] Jong-Sen Lee. 1980. Digital image enhancement and noise filtering by use of local statistics. IEEE Trans. on Pattern Analysis and Machine Vision. (March 1980), 165-168.

[9] F. Jin, P. Fifguth, L. Winger and E. Jernigan. 2003 Adaptive Wiener filtering of noisy images and image sequences. In Proceedings of IEEE International Conference on Image Processing. III-349-352.

[10] D.T. Kaun, A.A. Sawchuk, T.C. Strand and P. Chavel. 1985. Adaptive noise smoothing filter for images with signal dependent noise. IEEE Trans. on Pattern Analysis and Machine Vision, PAMI-7. (March 1985), 165-177.

[11] C. Tomasi and R. Manduchi. 1998. Bilateral filtering for gray and color images. In Proceedings of IEEE International Conference on Computer Vision, Bombay, India.

[12] Rafel C. Gonzalez and Richars E. Woods. 2009. Digital image processing. Pearson education.

[13] D. Barash 2002. A fundamental relationship between bilateral filtering, adaptive smoothing and the nonlinear diffusion equation. IEEE Trans. on Pattern Analysis and Machine Vision. (June 2002), 844-847.

[14] Wilbur C.K. Wong, Albert C.S. Chung and Simon C.H. Yu. 2004. Trilateral filtering for biomedical images. In Proceedings of IEEE International Symposium on Biomedical Imaging: Nano to Micro.

[15] L. Rudin and Stanley Osher. 1994. Total variation based image restoration with free local constraints. In Proceedings of IEEE International Conference on Image Processing, Austin Tx.

[16] Tony F. Chen, Stanley Osher and Jianhong Shen. 2001. The digital TV filter and nonlinear denoising. IEEE Trans. on Image Processing. (Feb. 2001), 231-241.

[17] Antoni Buades, Bartoneu Coll and Jean Michel Morel. 2004. On image denoising methods. SIAM Review.

[18] Antoni Buades, Bartoneu Coll and Jean Michel Morel. 2005. Image denoising by non-local averaging. In Proceedings of IEEE International Conference on Acoustics, Speech and Signal Processing.

[19] Antoni Buades, Bartoneu Coll and Jean Michel Morel. 2010. Image denoising methods, a new non-local principle. SIAM Review.
[20] Mona Mahmoudi and Guillerno Sapiro. 2005. Fast image and video denoising via non-local means of similar neighbourhoods. IEEE Signal Processing Letters. (Dec. 2005), 839-842.

[21] R. L. Kashyap and R. Chellappa. 1981. Filtering of noisy images using Markov random field models. In Proceedings of $19^{\text {th }}$ Allerton Conference on Communication Control and Computing, Urbana, Illinois.

[22] S. Geman and D. Geman. 1984. Stochastic relaxation, Gibbs distributions and the Bayesian restoration of images. IEEE Trans. on Pattern Analysis and Machine Intelligence. (Nov. 1984), 721-741.

[23] J. Besag. 1986. On the statistical analysis of dirty pictures. Journal of Royal Statistical Society, (1986), 259-302.

[24] J. Marroquin, S. Mitter, and T. Poggio. 1987 Probabilistic solutions of ill-posed problems in computational vision. Journal of American Statistical Association. (March 1987), 76-89.

[25] R. Szeliski. 1990. Bayesian modeling of uncertainty in low-level vision. International Journal on Computer Vision. (1990), 271-301.

[26] G. Sebastiani and F. Godtliebsen. 1997. On the use of Gibbs priors for Bayesian image restoration. Signal Processing. (Jan. 1997), 111-118.

[27] J. Sun, N.-N. Zhen, and H.-Y. Shum. 2003. Stereo matching using belief propagation. IEEE Trans. Pattern Analysis and Machine Intelligence. (2003), 787-800.

[28] F. Heitz and P. Bouthemy. 1993. Multimodal estimation of discontinuous optical flow using Markov random fields. IEEE Trans. on Pattern Analysis and Machine Intelligence. (Dec. 1993), 1217-1232.

[29] M. Varma and A. Zisserman. 2005. A statistical approach to texture classification from single images. International Journal of Computer Vision, (April 2005), 61-81.

[30] J. Besag. 1974. Spatial interaction and the statistical analysis of lattice system. Journal of the Royal Statistical Society, Series B. (1974), 192-236.

[31] K.V. Suresh, G. Mahesh kumar and A.N. Rajagopalan 2007. Super-resolution of license plates in real traffic videos. IEEE Trans. on Intelligent Transportation Systems. (June 2007), 321-331.

[32] S.Z. Li. 1995. Markov random field modeling in computer vision. Springer-Verlag.

[33] A. Blake and A. Zisserman. 1987. Visual reconstruction. MIT press.

[34] K. N. Chaudhury, D. Sage, and M. Unser. 2011. Fast $\mathrm{O}(1)$ bilateral filtering using trigonometric range kernels. IEEE Trans. on Image Processing. (June 2011), 33763382 .

[35] Zhou wang, Alan Conrad bovik, Hamid Rahim sheikh and Eero P. Simoncelli. 2004. Image quality assessment: From error visibility to structural similarity. IEEE Trans. on Image Processing. (April 2004), 600-612. 